\title{
Transmission Dynamics of Seasonal Influenza in Abidjan: Epidemiology and Modeling
}

\author{
Anderson K. N'gattia*1, 2 , Daouda Coulibaly ${ }^{1}$, Djibril Cherif ${ }^{1}$, Hervé Kadjo ${ }^{3}$ and Issaka \\ Tiembré ${ }^{1,2}$
}

'Epidemiologic surveillance service, Institut National d'Hygiène Publique, Abidjan, Côte d'Ivoire; 'Université Félix Houphouët-Boigny, Abidjan, Côte d'Ivoire; 'Institut Pasteur de Cote d'Ivoire, Abidjan, Côte d'Ivoire

\section{Objective}

This study aims to determine the epidemiological and clinical profiles of influenza infections related to different strains and the effect of climatological parameters on the temporal distribution of the disease for the prediction.

\section{Introduction}

Influenza poses a global health threat. The disease affects all ages, often with variable clinical features.

Abidjan, where this study took place, has a long rainy season AprilJuly with a shorter less intense rainy season October-November. Temperatures vary very little during the year. In temperate areas, children and adults aged $\geq 65$ years are risk groups. In these countries the seasonality of influenza is clearly defined, with seasonal epidemics in cold weather periods (1). But in the tropics, the risk groups of influenza are not as well defined. Also, the dynamics of influenza transmission and climatological parameters that influence it are specific to the tropical region and not as thoroughly studied.

\section{Methods}

Cote d'Ivoire's Institut National d'Hygiène Publique (INHP) operates sentinel influenza surveillance sites throughout the country, including Abidjan, following WHO guidelines. Staff at each site obtain specimens for influenza testing from a sample of the people treated each day for influenza-like illness (ILI) or severe acute respiratory infection (SARI). Data used in this report include all persons with laboratory-confirmed influenza from the sites located in Abidjan during 2007 to 2012, and climatological data from the SODEXAM (National Weather Service). The $\mathrm{X}^{2}$ test was used to compare proportions of cases by influenza type and also by month and season. Sequential logistic regression models were used to predict the epidemiological and clinical profiles, and multiple linear regression was used to assess the association between the number of weekly influenza cases and rainfall, relative humidity and ambient temperature. Explanatory models of weekly influenza cases based on climatological parameters used data from 2007 to 2010. Including data from 2011 to 2012 allowed us to test the prediction models. Modeling through the Box-Jenkins method was used with ARIMA process. Data were analyzed using Stata MP 12.0 software, StataCorp LP, College Station, Texas.

\section{Results}

A total of 921 influenza cases were identified over the period 2007-2010. Of these, $663(72 \%)$ were influenza A and $258(28 \%)$ were influenza B. Among the influenza A, $60(9.1 \%)$ were H1N1, 177 (26.7\%) seasonal H3N2, 34 (5.1\%) pH1N1 (2009 pandemic strain), and $392(59.1 \%)$ unsubtyped. The largest number of positive specimens was from young children aged 0-4 years (472; $52.9 \%$ ). Individuals with $\mathrm{pH} 1 \mathrm{~N} 1$ were more likely than those with other $\mathrm{H} 1 \mathrm{~N} 1$ to have cough $(\mathrm{OR}=10.52 ; 95 \% \mathrm{CI}=1.71$ to 64.57$)$ and more likely than those with influenza B to be from the age group of $5-14$ years $(\mathrm{OR}=3.10 ; 95 \% \mathrm{CI}=1.32$ to 7.3$)$. For patients with the $\mathrm{H} 3 \mathrm{~N} 2$ influenza, cough $(\mathrm{OR}=6.3 ; 95 \% \mathrm{CI}=2.466$ to 16.347$)$ and arthralgia/myalgia $(\mathrm{OR}=2.54 ; 95 \% \mathrm{CI}=1.08$ to 5.95$)$ were the best predictors versus influenza H1N1. The highest monthly and seasonally proportions of influenza viruses were observed the months corresponding to the long rainy season and the short rainy season in which respectively $48.4 \%$ and $20.2 \%$ of influenza viruses were isolated. The multiple linear regression showed association between the number of weekly influenza cases and rainfall $(\beta=0,025 ; \mathrm{p}=0.008)$ but not with relative humidity $(\mathrm{p}=0.108)$ and ambient temperature $(\mathrm{p}=0.786)$. ARIMAX models perform best only with the introduction of rainfall at lag (epidemiological weeks: $\mathrm{EW}) \mathrm{EW}_{0}(0.187)$ and $\mathrm{EW}_{-5}$ (0.175). The ARIMAX $(2,0,0) \mathrm{RF}$, was the best model (performance: $\mathrm{AIC}=1257.7$; $\mathrm{BIC}=1277.6$ ). The prediction of the weekly incidence of influenza from EW1(2011) to EW52 (2012) was implemented by ARIMAX $(2,0,0) \mathrm{RF}$ and fitted best values compared with observed values.

\section{Conclusions}

The specific epidemiological and clinical profiles of influenza were clearly identified in our study. Weekly rainfall was a good predictor of the transmission of seasonal influenza in Abidjan, in a setting with little change in temperature through the year. This should allow physicians to detect influenza cases and strengthen surveillance and preventive measures at the approach of rainy seasons.

\section{Keywords}

Transmission dynamics; seasonal influenza; epidemiological and clinical profiles; climatological parameters; Cote d'Ivoire

\section{References}

1. OMS | Grippe (saisonnière) [Internet]. WHO:www.who.int/ mediacentre/factsheets/fs211/fr/index.html

\author{
*Anderson K. N'gattia \\ E-mail: jeanandersonk@yahoo.fr
}

